\title{
Análise de Crescimento de ERVA-De-Touro em Competição Com Soja Cultivada sob Deficiência HídRICA ${ }^{1}$
}

\author{
Growth Analysis of Coatbutton in Competition with Soybean under Water Deficit
VIVIAN, R. ${ }^{2}$, DOURADO-NETO, D. ${ }^{3}$, SILVA, A.A. ${ }^{4}$, VICTORIA FILHO, R. ${ }^{5}$, YEDA, M.P. ${ }^{6} \mathbf{e}$ RUIZ-CORREA, S. ${ }^{7}$

\begin{abstract}
RESUMO - Devido à ausência na caracterização competitiva de espécies de plantas daninhas e suas habilidades sob condições edafoclimáticas desfavoráveis, avaliou-se o potencial de competição de Tridax procumbens em meio à cultura da soja. O cultivo foi realizado em condições controladas, em câmara de crescimento, utilizando-se o sistema de séries substitutivas, o qual abrangeu os monocultivos das espécies e a sua competição, com suprimento adequado de água e sob condições de deficiência hídrica temporária. Avaliaram-se a demanda evapotranspiratória, altura de planta, área foliar total e específica, razões de massa de matéria seca de raiz, caule e folha, taxas de assimilação e fotossintese líquida e a atividade de nitrato redutase aos 20, 50 e 70 dias após o transplante, bem como os teores de macronutrientes das espécies. Observaram-se menores demanda evapotranspiratória, área e duração foliar, assim como taxa de assimilação liquida de carbono, para T. procumbens, com consequente aumento da área foliar especifica e da razão de área foliar. Mesmo sob deficiência hídrica temporária, a soja apresentou maiores altura de planta, área foliar e massa de matéria seca total. A fotossintese liquida da soja foi superior à da planta daninha, embora esta tenha superado a cultura no início do seu cultivo. A atividade de nitrato redutase também foi maior para a soja em relação à planta daninha. Pelas análises dos minerais, T. procumbens demonstrou alteração na partição destes, principalmente com concentração de $\mathrm{K}$ e $\mathrm{Mg}$ em folhas e caules, respectivamente. Por meio das comparações entre os tratamentos, concluiu-se que $T$. procumbens apresenta baixo potencial competitivo quando em cultivo com a soja, mesmo sob condições de deficiência hídrica temporária. Não foi possivel distinguir completamente os efeitos da competição por água dos demais recursos do meio entre soja e $T$. procumbens, embora se verifique maior competição intraespecífica da cultura.
\end{abstract}

Palavras-chave: Glycine max, Tridax procumbens, fotossíntese, água.

\begin{abstract}
Due to the existence of poor competitive characterization of weeds and of their competitive ability under unfavourable climate and soil conditions, the competition potential of Tridax procumbens and soybean was evaluated. The experiment was carried out under control conditions in a growth chamber, using a replacement series system including the monoculture and its competing weeds, under normal water conditions and temporary water deficiency. The assessments included evapotranspiration, height, leaf and specific leaf area, dry matter ratios of root, stem, and leaf, carbon net assimilation and photosynthesis rate, and nitrate reductase activity at 20,50, and 70 days after the transplant, as well as macronutrient levels. Minor evapotranspiration, length, and leaf area, as well as carbon net assimilation rate of $\boldsymbol{T}$. procumbens were observed, with increases in specific leaf area and leaf area ratio. Even undertemporary water deficit, soybean showed higher height, leaf area, and total dry matter. The soybean's net assimilation rate was higher than that of the weed, although the latter surpassed the former at the beginning of the crop's growth. Nitrate
\end{abstract}

\footnotetext{
1 Recebido para publicação em 7.5.2012 e aprovado em 14.10.2012.

2 Pesquisador Embrapa Meio Norte, Av. Duque de Caxias, 5650, Buenos Aires, 64006-220 Teresina-PI, Brasil, <rafael.vivian@cpamn.embrapa.br>; ${ }^{3}$ Professor, Pesquisador ESALQ/USP, Piracicaba-SP, Dep. Produção Vegetal, $<$ durval.dourado@gmail.com>; ${ }^{4}$ Professor, Pesquisador, Dep. Produção Vegetal, Universidade Federal de Viçosa - DFT/UFV, Viçosa-MG, <aasilva@ufv.br>; ${ }^{5}$ Professor, Pesquisador, Dep. Produção Vegetal ESALQ/USP, Piracicaba-SP, <rvictori@esalq.usp.br>;

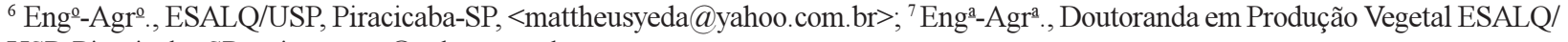
USP, Piracicaba-SP, <simonetrc@yahoo.com.br>
}

Planta Daninha, Viçosa-MG, v. 31, n. 3, p. 599-610, 2013 
reductase activity was also higher for the soybean. Mineral analysis showed changes in the weed's partition, mainly in $K$ and $M g$ concentration in the leaves and stems, respectively. Based on the comparisons of the treatments, it was concluded that $\boldsymbol{T}$. procumbens presents low competitive potential when grown with soybean, even under water deficit. It was not possible to distinguish the effect caused by competition for water from the other competitive effects between soybean and T. Procumbens, although a greater intra-specific competition by the crop was verified.

Keywords: Glycine max, Tridax procumbens, photosynthesis, water.

\section{INTRODUÇÃO}

O uso de herbicidas e a implementação de técnicas de controle de plantas daninhas têm proporcionado praticidade e rapidez na redução da sua interferência nas culturas. Entretanto, a ciência das plantas daninhas esteve, por muito tempo, focada em estudos relacionados a herbicidas, doses e períodos de controle, sem informações básicas da biologia e ecologia das plantas daninhas em seu agroecossistema. Ao mesmo tempo, durante o controle de determinada espécie, torna-se indispensável a atenção às demais espécies de menor ocorrência na área, as quais podem dominar o espaço livre biológico, tornando-se novo problema de controle (Mortensen et al., 2000).

A espécie Tridax procumbens tem sido utilizada como fonte alimentar (Singh et al., 2007) e estudada no tratamento medicinal em animais de outros países (Mohammed et al., 2008). No Brasil, porém, o aumento da sua ocorrência em áreas de cultivo de soja tem demandado maior atenção, sendo considerada planta daninha comum entre as regiões Sul e Centro-Oeste (Guimarães et al., 2004). Embora a maioria dos trabalhos com $T$. procumbens esteja relacionada com suas propriedades medicinais, essa espécie também está presente em áreas agrícolas de outros países, sendo responsável pela redução produtiva nas lavouras, como em soja (Jeyabal et al., 2001). A capacidade de disseminação das suas sementes pelo vento contribui para sua proliferação em diferentes áreas, dificultando o controle da espécie.

Nesse sentido, as análises dos parâmetros de crescimento de plantas daninhas, especialmente para $T$. procumbens, e sua relação competitiva com as culturas são de fundamental importância na determinação de táticas e no manejo dessa espécie, mesmo quando focado no uso de herbicidas (Erasmo et al., 1997; Christoffoleti, 2001). Mais recentemente, modelos baseados no acúmulo e na partição de massa de matéria seca das plantas também têm sido propostos e validados para algumas culturas, em razão da importância desse conhecimento na redução da competição e no aumento da produtividade (Baumann et al., 2001).

Em condições de estresse por adversidades climáticas, as análises dos componentes de produção de massa de matéria seca, área foliar e assimilação líquida de carbono tornam-se ainda mais importantes. O aumento da escassez hídrica nos últimos anos demanda maior atenção nos estudos de biologia e competição de plantas daninhas, pois a disponibilidade de água está diretamente relacionada às taxas de crescimento e eficiência competitiva de plantas por este e demais recursos.

Considerando necessária a avaliação da capacidade competitiva de Tridax procumbens junto à cultura da soja, o objetivo deste estudo foi avaliar as diferenças de resposta do monocultivo de cada espécie e da sua competição, sob condições normais de disponibilidade e/ou deficiência hídrica, no início de desenvolvimento das plantas.

\section{MATERIAL E MÉTODOS}

O experimento foi instalado em câmara de crescimento, modelo E15 (Conviron $\left.{ }^{\circledR}\right)$, com área e altura úteis de $1,4 \mathrm{~m}^{2}$ e $1,17 \mathrm{~m}$, respectivamente. As condições de temperatura e umidade relativa do ar foram controladas, respeitando as condições ótimas estabelecidas para a cultura da soja. As temperaturas permaneceram entre 25 e $30^{\circ} \mathrm{C}$ no período diurno e entre 18 e $22{ }^{\circ} \mathrm{C}$ durante o período noturno. $\mathrm{O}$ fotoperíodo estabelecido foi constante 
durante todo o ciclo da cultura, com 14 horas de luz e 10 horas na sua ausência. A radiação fotossinteticamente ativa (RFA) incidente foi de cerca de $800 \mu \mathrm{mol} \mathrm{m}^{-2} \mathbf{s}^{-1}$.

As sementes de Tridax procumbens foram adquiridas e pré-selecionadas para obter maior uniformidade. A soja cultivada foi de ciclo semiprecoce (BRS 232), de período juvenil longo. Sementes de $T$. procumbens e soja foram pré-germinadas, e as plântulas, imediatamente transferidas para vasos cilindricos de $4 \mathrm{~L}$ (15 cm de diâmetro x $30 \mathrm{~cm}$ de altura, com aproximadamente $140 \mathrm{~g}$ ). Utilizou-se como substrato Latossolo Vermelho-Amarelo previamente peneirado e com $\mathrm{pH}$ de 4,5. Por meio de sua análise química, constataram-se teores de $1,7 \%$ de matéria orgânica, $4,55 \mathrm{mg} \mathrm{dm}^{-3} \mathrm{de}$ $\mathrm{P}_{2} \mathrm{O}_{5}$ e 0,7, 11,0, 4,0, 3,0 e 28,0 $\mathrm{mmol}_{\mathrm{c}} \mathrm{dm}^{-3} \mathrm{de}$ $\mathrm{K}^{+}, \mathrm{Ca}^{+2}, \mathrm{Mg}^{+2}, \mathrm{Al}^{+3} \mathrm{e} \mathrm{H}^{++} \mathrm{Al}^{+3}$, respectivamente, com saturação por bases de $35,9 \%$. Foram cultivadas duas plantas por vaso, permanecendo sempre a mesma densidade, em monocultivo ou competição, sendo a massa total (vaso + solo + água + plântulas) aferida no momento do seu preenchimento.

A padronização do número de plantas por vaso baseou-se no sistema experimental de séries substitutivas, o qual permite distinguir qual das espécies é mais ou menos tolerante às variações impostas pelo ambiente, sendo avaliado neste estudo o efeito da deficiência hídrica (DH). Deve-se ressaltar que, em experimentos realizados em séries substitutivas, o aumento da população não interfere qualitativamente na dinâmica competitiva entre as espécies, ou seja, a dominância de uma espécie sobre a outra não se altera em função do tamanho da população (Cousens \& O'neill, 1993).

A adequação da saturação por bases foi realizada por meio da adição diária de solução nutritiva (Tabela 1). A redução do fornecimento de água ocorreu a partir dos 20 dias após o transplante (DAT), sendo mantida até 40 DAT. Os teores de água no solo foram diariamente monitorados pelo método de pesagem, sendo admitidos 50\% (com deficiência hídrica- CDH) e $100 \%$ (sem deficiência hídrica - SDH) da quantidade de água inicial. Para cada vaso contendo 4,4 kg de solo seco ao ar foi adicionado 0,900 L de água inicial, correspondendo à capacidade de saturação previamente determi-

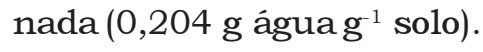

A partição de massa de matéria seca de raiz, caule, folha e fruto das espécies foi realizada aos 20, 50 e 70 DAT. Calcularam-se o índice de área foliar (IAF), a área foliar específica (AFE) e a razão de área foliar (RAF) a partir dos dados de área foliar (AF) (integrador de área LI-Cor LI 3100). Incluíram-se ainda as análises da taxa de assimilação líquida (TAL) e da duração de área foliar entre os intervalos de coleta (Tabela 2).

Utilizou-se analisador de trocas gasosas por infravermelho (LI-COR, LI-6400 XT) para avaliação da fotossintese líquida, condutância estomática e transpiração. As medições foram realizadas de acordo com os procedimentos padrões de calibração recomendados, aos 20, 50 e 70 DAT, entre 9 e 11 horas, com $800 \mu \mathrm{mol}$ fótons $\mathrm{m}^{-2} \mathrm{~s}^{-1}$ e $\Delta \mathrm{CO}_{2}+\Delta \mathrm{H}_{2} \mathrm{O}$ inferior a $1 \%$.

Utilizou-se a comparação da atividade da enzima nitrato redutase (NR) (EC 1.6.6.1.) para observar a capacidade de assimilação de nitrogênio entre as espécies e sua resposta em condições de deficiência hídrica, aos 20, 50 e 70 DAT. Realizou-se a medição indireta da sua atividade, estimando-se a concentração de $\mathrm{N}-\mathrm{NO}_{2}$ - presente na solução de $\mathrm{NO}_{3}{ }^{-}$incubada com discos foliares de soja e $T$. procumbens (Nicholas et al., 1976).

Tabela 1 - Composição da solução nutritiva utilizada no cultivo de soja e Tridax procumbens em monocultivo e em competição

\begin{tabular}{|c|c|c|c|c|c|}
\hline $\mathrm{MgSO}_{4}$ & $\mathrm{CaNO}_{3}$ & $\mathrm{KNO}_{3}$ & $\mathrm{MKP}$ & Fe-EDTA & Composto com micronutrientes \\
\hline \multicolumn{7}{|c|}{$\left(\mathrm{mg} \mathrm{L}^{-1}\right)$} & $\mathrm{B}(2 \%), \mathrm{Cu}(0,8 \%), \mathrm{Mo}(0,32 \%), \mathrm{Mn}(3,2 \%), \mathrm{Zn}(2,0 \%)$ \\
\hline 290 & 500 & 175 & 434 & 1,62 & $15,0 \mathrm{mg} \mathrm{L}^{-1}$ \\
\hline
\end{tabular}

Condutividade elétrica: $\pm 1,2 \mathrm{mS} \mathrm{cm}{ }^{-1}$. 
Tabela 2 - Representação das equações e unidades utilizadas nas análises de crescimento de soja e Tridax procumbens

\begin{tabular}{|c|c|}
\hline Parâmetro avaliado / Equação & Unidade \\
\hline Altura de planta & $\mathrm{cm}$ \\
\hline $\begin{array}{l}\text { Massa de matéria seca total }(\text { MMST) }=\mathrm{R}+\mathrm{C}+\mathrm{Fl}+\mathrm{F} \text {, em que } \mathrm{R}, \mathrm{C}, \mathrm{Fl} \text { e F representam a massa de matéria seca de } \\
\text { raiz, caule, folhas e frutos, respectivamente }\end{array}$ & $\mathrm{g}$ \\
\hline Razão de massa de matéria seca de raiz (RMMSR)=MMSR/MMST & $\mathrm{g} \mathrm{g}^{-1}$ \\
\hline Razão de massa de matéria seca de caule (RMMSC)=MMSC/MMST & $\mathrm{g} \mathrm{g}^{-1}$ \\
\hline Razão de massa de matéria seca de folha (RMMSFl)=MMSFl/MMST & $\mathrm{g} \mathrm{g}^{-1}$ \\
\hline Área foliar (AF) & $\mathrm{cm}^{2}$ \\
\hline 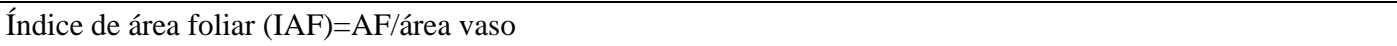 & - \\
\hline Área foliar específica $(\mathrm{AFE})=\mathrm{AF} / \mathrm{Fl}$ & $\mathrm{dm}^{2} \mathrm{~g}^{-1}$ \\
\hline Razão de área foliar (RAF)=AF/MMST & $\mathrm{dm}^{2} \mathrm{~g}^{-1}$ \\
\hline Duração de área foliar $(\mathrm{DAF})=\mathrm{IAF}_{\mathrm{i}}+\mathrm{IAF}_{\mathrm{i}-1} /(\Delta \mathrm{T} * 2)$, sendo $\Delta \mathrm{T}$ o intervalo, em dias, entre as avaliações & dias \\
\hline $\begin{array}{l}\text { Taxa de assimilação líquida }(\mathrm{TAL})=\left(\mathrm{MMST}_{2} / \mathrm{AF}_{2}-\mathrm{MMST}_{1} / \mathrm{AF}_{1}\right) *\left\{[\alpha /(\alpha-1)] /\left(\mathrm{T}_{2}-\mathrm{T}_{1}\right)\right\} \text {, em que } \alpha=\left(\ln \mathrm{MMST}_{2}\right. \\
\left.-\ln \mathrm{MMST}_{1}\right) /\left(\ln \mathrm{AF}_{2}-\ln \mathrm{AF}_{1}\right)\end{array}$ & $\mathrm{g} \mathrm{dm}^{-2} \mathrm{dia}^{-1}$ \\
\hline
\end{tabular}

Os teores de macronutrientes foram determinados em plantas aos 50 DAT. Após a coleta, as frações (raiz, caule e folhas) foram mantidas em estufa de ar ventilado a $65{ }^{\circ} \mathrm{C}$ durante 48 horas, para determinação da massa de matéria seca. As amostras foram trituradas em moinho do tipo Wiley com peneira de 20 mesh, com posterior extração por meio de digestão sulfúrica para as análises de nitrogênio $(\mathrm{N})$ e de digestão nitroperclórica para os demais minerais, de acordo com as técnicas descritas para cada nutriente (Malavolta et al., 1997).

Utilizou-se delineamento inteiramente casualizado, sendo os tratamentos compostos pela combinação dos três sistemas: soja (monocultivo), soja + planta daninha (competição) e planta daninha (monocultivo), com e sem deficiência hídrica, em esquema fatorial com 10 repetições. Todos os dados foram submetidos à análise da variância; quando significativos, utilizou-se o teste de Tukey a $5 \%$ de probabilidade de erro para comparação das médias. A comparação entre as médias dos tratamentos $\mathrm{CDH}$ e SDH foi feita pelo teste $\mathrm{t}(\mathrm{p} \leq 0,05)$. Quanto às análises dos resultados referentes à cultura e à planta daninha em competição, optou-se pela separação do esquema fatorial, representando melhor o objetivo proposto.

\section{RESULTADOS E DISCUSSÃO}

Verifica-se que soja e Tridax procumbens em competição apresentaram maior demanda evapotranspiratória em relação à planta daninha em monocultivo, aos 50 e 70 dias após o transplante (DAT) (Figura 1). Quando comparado ao monocultivo de soja, observa-se similaridade na evapotranspiração acumulada (ET)

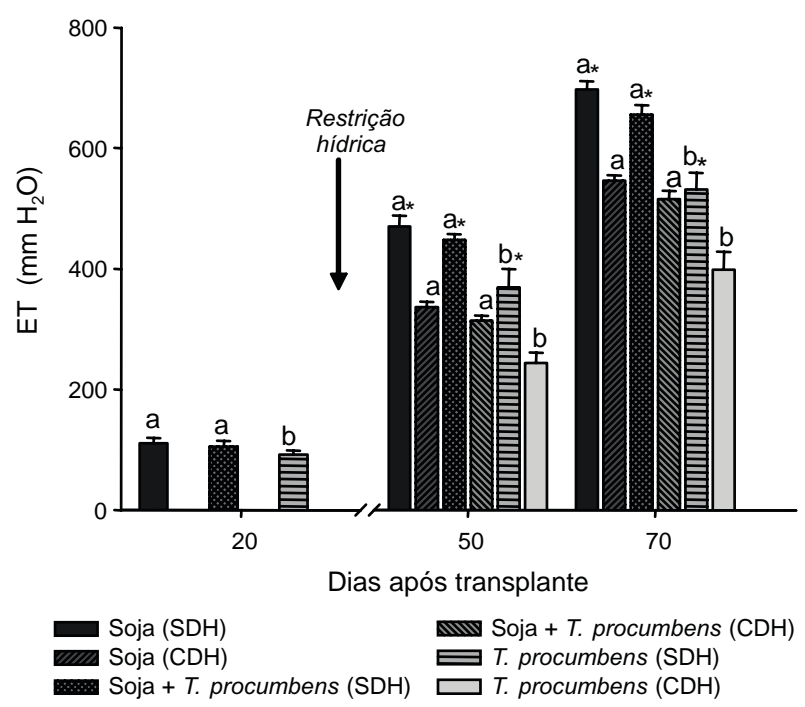

Colunas representando os tratamentos $\mathrm{SDH}$ ou $\mathrm{CDH}$ seguidas por uma mesma letra não diferem entre si pelo teste de Tukey $(\mathrm{p} \leq 0,05)$. * Diferença significativa entre os tratamentos $\mathrm{SDH}$ e $\mathrm{CDH}$, para cada composição de planta, pelo teste $\mathrm{t}(\mathrm{p} \leq 0,05)$.

Figura 1 - Evapotranspiração acumulada $\left(\mathrm{ET}-\mathrm{mm} \mathrm{H}_{2} \mathrm{O}\right)$ de soja e Tridax procumbens, em monocultivo ou competição, cultivadas em câmara de crescimento em condições normais de disponibilidade hídrica (SDH), aos 20 dias após o transplante (DAT), ou após a deficiência hídrica (CDH) temporária, aos 50 e 70 DAT. 
entre os tratamentos, atribuindo-se à soja o maior consumo de água em relação à planta daninha. A menor demanda evapotranspiratória da planta daninha está de acordo com os valores de massa de matéria seca total (MMST) e área foliar (AF), demonstrados posteriormente para $T$. procumbens.

As variáveis altura e MMST indicaram que a soja possui maior desenvolvimento inicial que $T$. procumbens, conferindo vantagens competitivas para a cultura em relação à planta daninha (Tabela 3). A diferença de altura entre as espécies no início do seu crescimento permite o estabelecimento rápido da cultura e, consequentemente, maior interceptação da radiação luminosa.

As análises comparativas entre os tratamentos com $(\mathrm{CDH})$ e sem deficiência hídrica (SDH), para cada composição de planta, comprovam que a $\mathrm{DH}$ não interferiu na altura das plantas $(p \leq 0,05)$, embora o seu efeito tenha minimizado as diferenças de MMST entre soja e T. procumbens (Tabela 3). A MMST da planta daninha em competição com a cultura e sob DH foi reduzida em média $22 \%$ em relação à ausência da deficiência. Todavia, a redução para a soja foi maior, com cerca de $42 \%$ da MMST obtida no tratamento sem DH. Em ambos

Tabela 3 - Variáveis de crescimento e alocação de fitomassa de soja e T. procumbens em monocultivo ou em competição (+), cultivadas em câmara de crescimento e submetidas ou não à deficiência hídrica temporária

\begin{tabular}{|c|c|c|c|c|c|c|}
\hline \multirow{3}{*}{ Composição de plantas } & \multirow{3}{*}{$\begin{array}{l}\text { Restrição } \\
\text { hídrica } 1 /\end{array}$} & \multicolumn{5}{|c|}{20 DAT } \\
\hline & & Altura & MMST & RMMSR & RMMSC & RMMSFl \\
\hline & & $(\mathrm{cm})$ & (g por planta) & \multicolumn{3}{|c|}{$\left(\mathrm{g} \mathrm{g}^{-1}\right)$} \\
\hline Soja & \multirow{4}{*}{ SDH } & $25,60 \mathrm{a}$ & $1,43 \mathrm{~b}$ & $0,22 \mathrm{c}$ & $0,30 \mathrm{a}$ & $0,49 \mathrm{a}$ \\
\hline Soja + & & $24,10 \mathrm{a}$ & $2,31 \mathrm{a}$ & $0,27 \mathrm{~b}$ & $0,25 \mathrm{ab}$ & $0,48 \mathrm{a}$ \\
\hline T. procumbens + & & $10,10 \mathrm{~b}$ & $1,54 \mathrm{~b}$ & $0,35 \mathrm{a}$ & $0,21 \mathrm{~b}$ & $0,45 \mathrm{a}$ \\
\hline T. procumbens & & $8,75 \mathrm{~b}$ & $1,03 \mathrm{c}$ & $0,24 \mathrm{bc}$ & $0,26 a b$ & $0,50 \mathrm{a}$ \\
\hline & & \multicolumn{5}{|c|}{50 DAT } \\
\hline Soja & \multirow{4}{*}{ SDH } & $102,50 \mathrm{a}$ & $12,04 \mathrm{a}$ & $0,34 \mathrm{a}$ & $0,30 \mathrm{~b}$ & $0,37 \mathrm{ab}$ \\
\hline Soja + & & $102,20 \mathrm{a}$ & $12,39 \mathrm{a}$ & $0,15 \mathrm{~b}$ & $0,37 \mathrm{ab}$ & $0,48 \mathrm{a}$ \\
\hline T. procumbens + & & $60,50 \mathrm{~b}$ & $2,76 \mathrm{~b}$ & $0,09 \mathrm{~b}$ & $0,53 \mathrm{a}$ & $0,37 \mathrm{ab}$ \\
\hline T. procumbens & & $83,50 \mathrm{ab}$ & $6,03 \mathrm{~b}$ & $0,12 \mathrm{~b}$ & $0,53 \mathrm{a}$ & $0,28 \mathrm{~b}^{*}$ \\
\hline Soja & \multirow{4}{*}{$\mathrm{CDH}$} & $95,50 \mathrm{a}$ & $8,47 \mathrm{a}$ & $0,32 \mathrm{a}$ & $0,30 \mathrm{a}$ & $0,39 \mathrm{a}$ \\
\hline Soja + & & $99,00 \mathrm{a}$ & $8,73 \mathrm{a}$ & $0,14 a b$ & $0,39 \mathrm{a}$ & $0,46 \mathrm{a}$ \\
\hline T. procumbens + & & $60,00 \mathrm{~b}$ & $2,26 \mathrm{~b}$ & $0,07 \mathrm{~b}$ & $0,48 \mathrm{a}$ & $0,44 \mathrm{a}$ \\
\hline T. procumbens & & $85,00 \mathrm{ab}$ & $3,74 \mathrm{ab}$ & $0,13 \mathrm{ab}$ & 0,49 a & $0,36 \mathrm{a}$ \\
\hline & & \multicolumn{5}{|c|}{70 DAT } \\
\hline Soja & \multirow{4}{*}{ SDH } & $98,00 \mathrm{a}$ & $14,59 \mathrm{~b}$ & $0,14 \mathrm{a}$ & $0,26 \mathrm{~b}$ & $0,38 \mathrm{a}$ \\
\hline Soja + & & $104,00 \mathrm{a}$ & $22,16 \mathrm{a}$ & $0,11 a b$ & $0,27 \mathrm{~b}$ & $0,37 \mathrm{a}$ \\
\hline T. procumbens + & & $59,50 \mathrm{~b}$ & $3,11 \mathrm{~d}$ & $0,06 \mathrm{c}$ & $0,55 \mathrm{a}$ & $0,40 \mathrm{a}$ \\
\hline T. procumbens & & $96,40 \mathrm{ab}$ & $8,82 \mathrm{c}$ & $0,08 \mathrm{bc}$ & $0,56 \mathrm{a}$ & $0,33 \mathrm{a}$ \\
\hline Soja & \multirow{4}{*}{$\mathrm{CDH}$} & $103,50 \mathrm{a}$ & $15,17 \mathrm{a}$ & $0,07 \mathrm{a}$ & $0,22 \mathrm{~b}$ & $0,43 \mathrm{a}$ \\
\hline Soja + & & $97,00 \mathrm{a}$ & $17,57 \mathrm{a}$ & $0,10 \mathrm{a}$ & $0,27 \mathrm{~b}$ & $0,35 \mathrm{a}$ \\
\hline T. procumbens + & & $45,00 \mathrm{~b}$ & $2,08 \mathrm{~b}$ & $0,11 \mathrm{a}$ & $0,49 \mathrm{a}$ & $0,40 \mathrm{a}$ \\
\hline T. procumbens & & $98,50 \mathrm{a}$ & $5,38 \mathrm{~b}$ & $0,11 \mathrm{a}$ & $0,51 \mathrm{~b}$ & $0,37 \mathrm{a}$ \\
\hline
\end{tabular}

1/ SDH - sem deficiência hídrica; CDH - com deficiência hídrica entre 20 e 40 dias após o transplante (DAT); MMST - massa de matéria seca total; RMMSR - razão de massa de matéria seca de raiz; RMMSC - razão de massa de matéria seca de caule; RMMSFl - razão de massa de matéria seca de folha. Médias na coluna, dentro de cada período amostral (20,50 e $70 \mathrm{DAT})$, seguidas por uma mesma letra não diferem entre si pelo teste de Tukey $(\mathrm{p} \leq 0,05)$. * Diferença significativa entre os tratamentos SDH e CDH, para cada composição de planta, pelo teste $\mathrm{t}(\mathrm{p} \leq 0,05)$. 
os casos, as diferenças entre os tratamentos com e sem DH não foram estatisticamente comprovadas $(p \leq 0,05)$. Por meio das comparações de MMST aos 20 e 70 DAT, verifica-se ainda que o efeito competitivo intraespecífico da cultura foi maior em relação ao interespecífico.

De acordo com as razões de massa de matéria seca de raiz (RMMSR), caule (RMMSC) e folhas (RMMSFl), observa-se que a planta daninha em competição investe, inicialmente, mais fotoassimilados para a formação do seu sistema radicular (Tabela 3). Entretanto, aos 50 e 70 DAT, constata-se o aumento na porção de caule de $T$. procumbens, representando cerca de $50 \%$ da massa de matéria seca da planta daninha, tanto em competição como em monocultivo. Por ser a maior fração da planta, admite-se que o efeito competitivo de $T$. procumbens também está associado à incorporação de fotoassimilados ao sistema caulinar, o qual permite a reprodução desta planta daninha a partir da emissão de novas raízes quando em contato com o solo. Verifica-se ainda que a DH não interferiu na distribuição da massa de matéria seca da cultura e da planta daninha, tanto em condições de monocultivo como em competição, com exceção da RMMSF1 de $T$. procumbens em monocultivo aos 50 DAT.

Em relação ao efeito competitivo, verificase que, na ausência da planta daninha, a relação entre a massa de matéria seca da parte aérea e raiz (A/R) para soja é constante até os 50 DAT (Figura 2A), com aumento na incorporação de fotoassimilados para a parte aérea a partir do início do florescimento (50 DAT), decorrente do início da sua formação de grãos. Contudo, em competição com $T$. procumbens, verifica-se o aumento linear da relação $A / R$ em soja (Figura 2B), com priorização do acúmulo de seus fotoassimilados na parte aérea da cultura. Para T. procumbens, verifica-se ainda que as relações $A / R$, tanto em competição (Figura 2C) como em monocultivo (Figura 2D), são crescentes desde o período inicial, como resposta à alta capacidade competitiva de soja por luz.

Conforme o modelo de partição de Brouwer (1983), as plantas investem grande parte de seus fotoassimilados na porção menos favorecida ou sob maior competição. Portanto, as raízes têm seu crescimento limitado pelo suprimento de carbono das folhas, enquanto as folhas reduzem o crescimento pela limitação de água e nutrientes fornecidos pelas raízes. $O$ efeito da $\mathrm{DH}$ na relação $A / R$ parece pouco evidente, embora se saiba que a limitação hídrica interfere geralmente na porção superior das plantas, não comprometendo o sistema radicular sob curto período de submissão à DH (Gowing et al., 1990; Sharp, 2002).

A elevada competição da parte aérea de soja com a planta daninha pode ser evidenciada nas diferenças $(p \leq 0,05)$ em área foliar (AF) e IAF entre a cultura e $T$. procumbens cultivadas no mesmo vaso (Tabela 4). Todavia, verifica-se que $T$. procumbens tende a aumentar a sua capacidade de competição pelo incremento da área foliar específica (AFE) e da razão de área foliar (RAF). Segundo Feng et al. (2004), a AFE está inversamente correlacionada com os custos de formação de massa de matéria seca das folhas; os maiores valores verificados para $T$. procumbens aos 50 e 70 DAT podem auxiliar na competição da espécie, principalmente sob alta restrição luminosa.

Plantas com elevadas taxas de saturação fotossintética, de RMMSFl e de AFE apresentam vantagens competitivas sobre as demais espécies, com valores inferiores para essas variáveis (Feng et al., 2007). Comparativamente à soja, $T$. procumbens apresentou maior AFE e RAF (Tabela 4), similar RMMSFl aos 70 DAT e menor duração de área foliar (DAF) durante o ciclo de cultivo. O efeito negativo do sombreamento na DAF é constantemente relatado nos experimentos de competição (Patterson, 1982) ou em estudos de aclimatação de plantas (Juraimi et al., 2004), possuindo efeito direto no rendimento de grãos (Kumudini et al., 2001).

A taxa de assimilação líquida (TAL) da cultura foi aparentemente maior que a da planta daninha, embora com diferença estatística $(p \leq 0,05)$ somente para o tratamento em competição, aos 70 DAT (Tabela 4). Plantas com alta TAL e/ou elevada RAF tendem a apresentar rápido crescimento inicial, com melhor aproveitamento do espaço e interceptação da radiação luminosa. Comparativamente, tanto a cultura como a planta daninha apresentaram vantagens competitivas relacionadas à parte aérea. Contudo, a significativa diferença $(\mathrm{p} \leq 0,05)$ de $\mathrm{AF}$ entre as espécies foi responsável pela supressão de $T$. procumbens. 

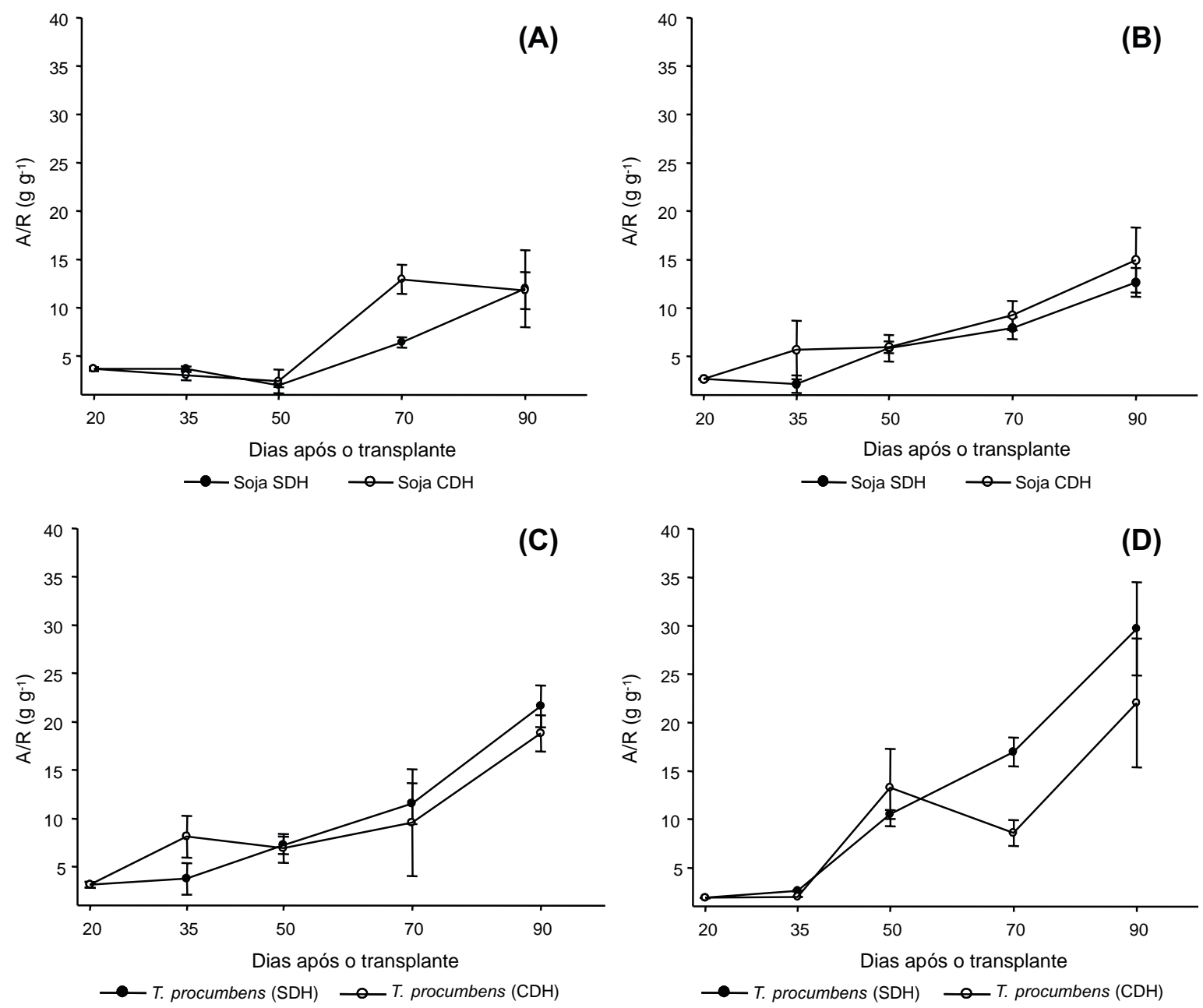

Figura 2 - Relação entre a massa de matéria seca da parte aérea e raiz (A/R) de soja e Tridax procumbens em monocultivo ou competição $(+)$, cultivadas em câmara de crescimento e submetidas ou não à deficiência hídrica temporária. (A) soja em monocultivo; (B) soja em competição; (C) T. procumbens em monocultivo; (D) T. procumbens em competição.

Apesar da maior taxa de fotossintese líquida (FL) de $T$. procumbens aos 20 DAT, a cultura conseguiu compensar sua menor assimilação fotossintética inicial ao longo do cultivo, com valores de FL superiores aos da planta daninha aos 50 e 70 DAT (Figura 3). Para a planta daninha em competição, o efeito da $\mathrm{DH}$ sobre a FL manteve-se aos 50 e 70 DAT, embora resultante também das demais vantagens competitivas relatadas para a cultura, pois não se observaram reduções significativas para a condutância estomática $(p \leq 0,05)$ nesse mesmo período (dados não apresentados). Os valores médios mensurados de condutância estomática para soja foram de 0,07 e $0,14 \mathrm{~mol} \mathrm{~m}^{-2} \mathbf{s}^{-1}$, quando em monocultivo e competição, respectivamente, sendo similares aos de $T$. procumbens, com médias comparativas de 0,07 e $0,12 \mathrm{~mol} \mathrm{~m}^{-2} \mathbf{s}^{-1}$. Portanto, acredita-se que as elevadas taxas de FL, juntamente com o rápido desenvolvimento inicial da soja e maior DAF, garantiram sua elevada TAL, com maior MMST ao final do ciclo de cultivo.

Verifica-se também maior $(\mathrm{p} \leq 0,05)$ atividade da enzima nitrato redutase (NR) para a soja em relação a $T$. procumbens (Figura 4), aos 20, 50 e 70 DAT. Sabe-se que a expressão da NR, assim como o ajuste da sua atividade, estão vinculados a variações ambientais e metabólicas. A intensidade luminosa está 
Tabela 4 - Parâmetros de crescimento foliar e de assimilação líquida de carbono de soja e Tridax procumbens em monocultivo ou em competição (+), cultivadas em câmara de crescimento e submetidas ou não à deficiência hídrica temporária

\begin{tabular}{|c|c|c|c|c|c|c|c|}
\hline \multirow{3}{*}{$\begin{array}{l}\text { Composição de } \\
\text { plantas }\end{array}$} & \multirow{3}{*}{$\begin{array}{l}\text { Restrição } \\
\text { hídrica } 1 \text { - }\end{array}$} & \multicolumn{6}{|c|}{20 DAT } \\
\hline & & \multirow{2}{*}{$\frac{\mathrm{AF}}{\left(\mathrm{cm}^{2}\right)}$} & \multirow{2}{*}{ IAF } & \multicolumn{2}{|c|}{ AFE } & \multicolumn{2}{|c|}{ RAF } \\
\hline & & & & \multicolumn{4}{|c|}{$\left(\mathrm{dm}^{2} \mathrm{~g}^{-1}\right)$} \\
\hline Soja & \multirow{4}{*}{ SDH } & $147,03 \mathrm{~b}$ & $1,66 \mathrm{~b}$ & \multicolumn{2}{|c|}{$210,02 \mathrm{ab}$} & \multicolumn{2}{|c|}{$102,68 \mathrm{ab}$} \\
\hline Soja + & & $367,42 \mathrm{a}$ & $4,16 \mathrm{a}$ & \multicolumn{2}{|c|}{$334,45 \mathrm{a}$} & \multicolumn{2}{|c|}{$159,72 \mathrm{a}$} \\
\hline T. procumbens + & & $101,28 \mathrm{~b}$ & $1,15 \mathrm{~b}$ & \multicolumn{2}{|c|}{$146,89 \mathrm{~b}$} & \multicolumn{2}{|c|}{$65,77 \mathrm{~b}$} \\
\hline \multirow[t]{4}{*}{ T. procumbens } & & $106,96 \mathrm{~b}$ & $1,21 \mathrm{~b}$ & \multicolumn{2}{|c|}{$210,38 \mathrm{ab}$} & \multicolumn{2}{|c|}{$103,81 \mathrm{ab}$} \\
\hline & & \multicolumn{6}{|c|}{ 50 DAT } \\
\hline & & $\mathrm{AF}$ & & AFE & RAF & \multirow{2}{*}{$\begin{array}{l}\text { DAF } \\
\text { (dias) }\end{array}$} & \multirow{2}{*}{$\begin{array}{c}\text { TAL } \\
\left(\mathrm{g} \mathrm{dm}^{-2} \mathrm{dia}^{-1}\right)\end{array}$} \\
\hline & & $\left(\mathrm{cm}^{2}\right)$ & $\mathrm{IAF}$ & \multicolumn{2}{|c|}{$\left(\mathrm{dm}^{2} \mathrm{~g}^{-1}\right)$} & & \\
\hline Soja & \multirow{4}{*}{$\mathrm{SDH}$} & $1102,64 \mathrm{~b}$ & $12,48 \mathrm{~b}$ & $248,05 \mathrm{~b}$ & $91,63 \mathrm{~b}$ & $0,23 \mathrm{~b}$ & $0,070 \mathrm{a}$ \\
\hline Soja + & & $1372,15 \mathrm{a}$ & $15,53 \mathrm{a}$ & $232,32 \mathrm{~b}$ & $111,38 \mathrm{ab}$ & $0,33 \mathrm{a}$ & $0,045 \mathrm{ab}$ \\
\hline T. procumbens + & & $506,93 \mathrm{c}$ & $5,74 \mathrm{c}$ & $502,09 \mathrm{a}$ & $183,73 \mathrm{a}$ & $0,11 \mathrm{c}$ & $0,020 \mathrm{~b}$ \\
\hline T. procumbens & & $923,10 \mathrm{~b}^{*}$ & $10,45 b^{*}$ & $597,31 \mathrm{a}$ & $157,74 \mathrm{ab}$ & $0,19 \mathrm{~b}^{*}$ & $0,050 \mathrm{ab}$ \\
\hline Soja & \multirow{4}{*}{$\mathrm{CDH}$} & $834,69 \mathrm{ab}$ & $9,45 \mathrm{ab}$ & $261,09 \mathrm{a}$ & $102,12 \mathrm{a}$ & $0,18 \mathrm{ab}$ & $0,060 \mathrm{a}$ \\
\hline Soja + & & $1177,42 \mathrm{a}$ & $13,33 \mathrm{a}$ & $293,82 \mathrm{a}$ & $136,32 \mathrm{a}$ & $0,29 \mathrm{a}$ & $0,030 \mathrm{ab}$ \\
\hline T. procumbens + & & $438,22 \mathrm{~b}$ & $4,96 \mathrm{~b}$ & $454,06 \mathrm{a}$ & $193,20 \mathrm{a}$ & $0,10 \mathrm{~b}$ & $0,015 \mathrm{~b}$ \\
\hline T. procumbens & & $656,72 \mathrm{ab}$ & $7,43 \mathrm{ab}$ & $530,26 \mathrm{a}$ & $180,39 \mathrm{a}$ & $0,14 \mathrm{ab}$ & $0,030 \mathrm{ab}$ \\
\hline & & \multicolumn{6}{|c|}{ 70 DAT } \\
\hline & & $\mathrm{AF}$ & & AFE & RAF & \multirow{2}{*}{$\begin{array}{l}\text { DAF } \\
\text { (dias) }\end{array}$} & \multirow{2}{*}{$\begin{array}{c}\text { TAL } \\
\left(\mathrm{g} \mathrm{dm}^{-2} \mathrm{dia}^{-1}\right)\end{array}$} \\
\hline & & $\left(\mathrm{cm}^{2}\right)$ & $\mathrm{IAF}$ & \multicolumn{2}{|c|}{$\left(\mathrm{dm}^{2} \mathrm{~g}^{-1}\right)$} & & \\
\hline Soja & \multirow{4}{*}{$\mathrm{SDH}$} & $975,29 \mathrm{bc}$ & $11,04 \mathrm{bc}$ & $178,49 b^{*}$ & $66,81 \mathrm{~b}$ & $0,58 \mathrm{~b}$ & $0,010 \mathrm{~b}^{*}$ \\
\hline Soja + & & $1347,99 \mathrm{ab}$ & $15,26 \mathrm{ab}$ & $162,65 \mathrm{~b}$ & $60,79 \mathrm{~b}$ & $0,77 \mathrm{a}$ & $0,035 \mathrm{a}$ \\
\hline T. procumbens + & & $639,02 \mathrm{c}$ & $7,23 \mathrm{c}$ & $523,22 \mathrm{a}$ & $208,98 \mathrm{a}$ & $0,32 \mathrm{c}$ & $0,005 \mathrm{~b}$ \\
\hline T. procumbens & & $1400,42 \mathrm{a}^{*}$ & $15,85 \mathrm{a}^{*}$ & $488,50 \mathrm{a}$ & $158,83 \mathrm{a}$ & $0,65 b^{*}$ & $0,015 \mathrm{ab}$ \\
\hline Soja & \multirow{4}{*}{$\mathrm{CDH}$} & $1068,72 \mathrm{ab}$ & $12,10 \mathrm{ab}$ & $132,31 \mathrm{~b}$ & $70,67 \mathrm{~b}$ & $0,53 \mathrm{~b}$ & $0,040 \mathrm{a}$ \\
\hline Soja + & & $1571,95 \mathrm{a}$ & $17,79 \mathrm{a}$ & $256,07 \mathrm{~b}$ & $89,53 \mathrm{~b}$ & $0,77 \mathrm{a}$ & $0,032 \mathrm{a}$ \\
\hline T. procumbens + & & $540,82 \mathrm{~b}$ & $6,12 b$ & $696,42 \mathrm{a}$ & $260,16 \mathrm{a}$ & $0,27 \mathrm{c}$ & $0,002 \mathrm{~b}$ \\
\hline T. procumbens & & $1118,00 \mathrm{a}$ & $12,65 \mathrm{a}$ & $651,95 \mathrm{a}$ & $233,58 \mathrm{a}$ & $0,50 \mathrm{~b}$ & $0,000 \mathrm{ab}$ \\
\hline
\end{tabular}

$\stackrel{1}{ }$ SDH - sem deficiência hídrica; CDH - com deficiência hídrica temporária entre 20 e 40 dias após o transplante (DAT). Médias seguidas por uma mesma letra na coluna (comparações entre tratamentos $\mathrm{SDH}$ ou $\mathrm{CDH}$ ) não diferem entre si pelo teste de $\mathrm{Tukey}(\mathrm{p} \leq 0,05$ ) * Diferença significativa entre os tratamentos SDH e CDH, para cada composição de plantas, pelo teste $t(p<0,10)$. ** Diferença significativa pelo teste de Tukey $(\mathrm{p}<0,10)$. AF - área foliar; IAF - índice de área foliar; AFE - área foliar específica; RAF - razão de área foliar; DAF - duração de área foliar, calculada para os intervalos 20-50 e 50-70 DAT; TAL - taxa de assimilação líquida.

diretamente envolvida na modulação da NR transcrita (Pereira-Netto et al., 1998; Oliveira, 2005), podendo reduzir a sua atividade na planta daninha. Assim, considerando o crescente sombreamento de $T$. procumbens pela cultura, admite-se o seu efeito na atividade da NR.

Com a restrição de água, a NR foi menor $(p \leq 0,05)$ para a soja mantida em monocultivo ou em competição com $T$. procumbens, não se observando a manutenção dos efeitos para a planta daninha. Portanto, a competição interespecífica não influenciou a atividade enzimática da cultura. Conforme Plhak (2003), a redução da NR sob DH está relacionada à menor transpiração das plantas, com consequente redução no influxo de nitrato para as folhas, o qual regula a necessidade de ativação da NR - fato esse evidenciado pelas respostas experimentais obtidas.

As análises de macronutrientes revelam que a soja tende a apresentar maiores $(p \leq 0,05)$ teores de $\mathrm{N}$ e $\mathrm{P}$, em relação a $T$. procumbens, para as porções de raiz, caule e folha (Tabela 5), principalmente em monocultivo. Entretanto, verificaram-se concentrações elevadas de $\mathrm{K}$, Ca e $\mathrm{Mg}$ em caule e de Ca e $\mathrm{Mg}$ em folhas da planta daninha. Sob competição com soja, os teores particionados de $\mathrm{S}$ e $\mathrm{Mg}$ em $T$. procumbens e na cultura foram similares.

Variações significativas $(p \leq 0,05)$ entre os teores de macronutrientes da planta daninha foram observadas quando esta passou por 


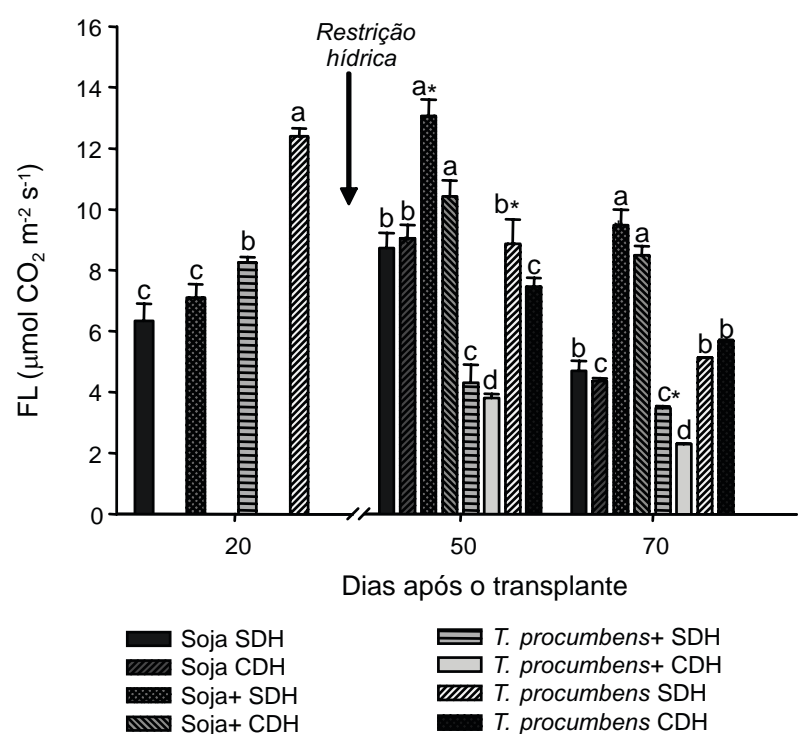

Colunas representando os tratamentos $\mathrm{SDH}$ ou $\mathrm{CDH}$, seguidas por uma mesma letra, não diferem entre si pelo teste de Tukey $(\mathrm{p} \leq 0,05)$. * Diferença significativa entre os tratamentos SDH e CDH, para cada composição de planta, pelo teste $\mathrm{t}(\mathrm{p} \leq 0,05)$.

Figura 3 - Fotossíntese líquida (FL) em amostras foliares de soja e Tridax procumbens em monocultivo ou competição $(+)$, em condições normais de disponibilidade hídrica (20 dias após o transplante - DAT) ou após deficiência hídrica temporária (50 e 70 DAT), estabelecida entre 20 e 40 DAT.

período de restrição por água em competição com a soja. Foram verificados incrementos nos teores de $\mathrm{P}$ e $\mathrm{K}$ foliares, assim como nos de $\mathrm{S}$, Ca e Mg, na porção caulinar de $T$. procumbens. Considerando que as concentrações totais de macronutrientes para $T$. procumbens não diferiram entre os tratamentos com ou sem deficiência hídrica, tanto em competição como em monocultivo, e que não foram constatadas variações significativas na massa de matéria seca total da planta daninha para esses tratamentos, admite-se que as diferenças específicas nos teores de $\mathrm{S}$, Ca e $\mathrm{Mg}$ em caule e de $\mathrm{P}$ e K em folhas são decorrentes do efeito competitivo com a cultura.

Em relação aos elevados teores de $\mathrm{K}$ em folhas, sabe-se que esse mineral desempenha importante função na manutenção do potencial osmótico celular, regulando a abertura e o fechamento estomático, o que poderia auxiliar na regulação da condutância estomática sob condições de baixa disponibilidade hídrica. $\mathrm{O}$ acúmulo de $\mathrm{Mg}$ em Ulmus pumila, por
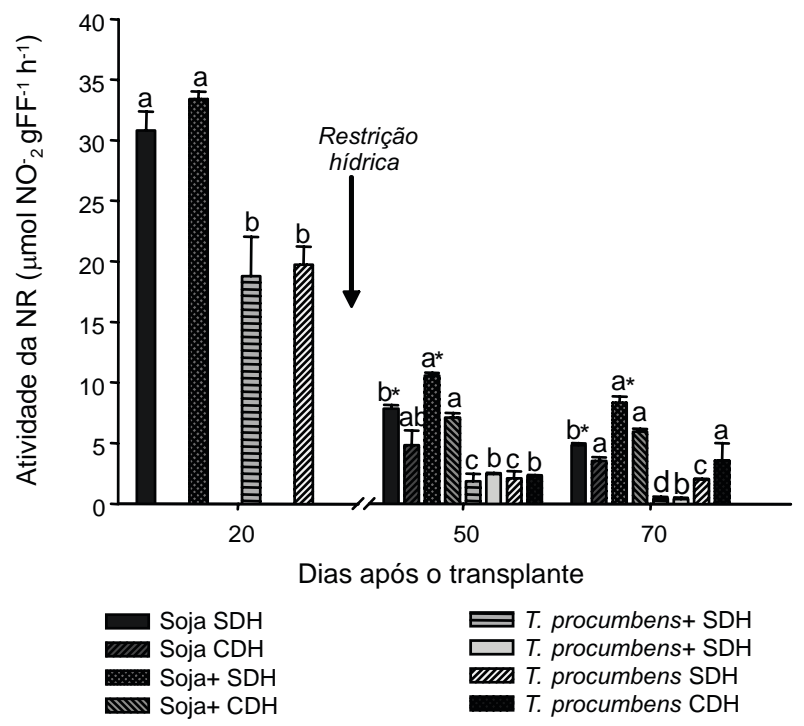

Colunas representando os tratamentos $\mathrm{SDH}$ ou $\mathrm{CDH}$, seguidas por uma mesma letra, não diferem entre si pelo teste de Tukey $(\mathrm{p} \leq 0,05)$. * Diferença significativa entre os tratamentos SDH e $\mathrm{CDH}$, dentro de cada composição de planta, pelo teste $t(p \leq 0,05)$.

Figura 4 - Atividade da enzima nitrato redutase (NR $\mu \mathrm{mol}\left(\mathrm{NO}_{2}^{-}\right) \mathrm{g}^{-1}$ massa de matéria fresca $\left.\mathrm{h}^{-1}\right)$ em amostras foliares de soja e Tridax procumbens em monocultivo ou competição $(+)$, em condições normais de disponibilidade hídrica (20 DAT) ou após deficiência hídrica temporária (50 e 70 DAT), estabelecida entre 20 e 40 DAT.

exemplo, foi relatado por Bosu \& Wagner (2007) em plantas submetidas à deficiência hídrica, as quais apresentaram maior tolerância à deficiência. González et al. (2002) também constataram aumento da concentração de $\mathrm{K}$, $\mathrm{Mg}$ e $\mathrm{Cl}$ em estipulas de Pisum sativum, com aumento do potencial osmótico. Para os incrementos nos teores de $\mathrm{P}$ e $\mathrm{S}$ em raiz, assim como no de $\mathrm{Ca}$ em caule, não foram encontrados relatos que auxiliassem na justificativa do ocorrido, reconhecendo-se a necessidade de maior aprofundamento nesses estudos.

Considerando o conjunto de variáveis analisadas, verifica-se que a espécie $T$. procumbens possui características competitivas distintas em relação à soja, incluindo a sua resposta à deficiência hídrica. Apesar da menor demanda evapotranspiratória, a planta daninha apresentou reduções na área e na duração de área foliar, assim como no IAF, quando se restringiu a disponibilidade de água em monocultivo. Variações também na distribuição de macronutrientes, principalmente $\mathrm{K} \mathrm{e} \mathrm{Mg}$, em caule e 
folhas ressaltam a adaptação de $T$. procumbens sob condições de competição e de deficiência hídrica.
Admite-se que as variações de resposta entre os tratamentos $\mathrm{CDH}$ e SDH também foram influenciadas pela competição com a

Tabela 5 - Teores de macronutrientes particionados em raiz, caule e folha $\left(\mathrm{g} \mathrm{kg}^{-1}\right)$ e totais $\left(\mathrm{mg} \mathrm{planta}^{-1}\right)$ em soja e Tridax procumbens em monocultivo ou em competição $(+)$, cultivadas em câmara de crescimento e submetidas ou não à deficiência hídrica temporária

\begin{tabular}{|c|c|c|c|c|c|c|c|}
\hline \multirow{3}{*}{$\begin{array}{l}\text { Composição de } \\
\text { plantas }\end{array}$} & \multirow{3}{*}{$\begin{array}{l}\text { Restrição } \\
\text { hídrica }^{\underline{1}}\end{array}$} & $\mathrm{~N}$ & $\mathrm{P}$ & $\mathrm{K}$ & $\mathrm{S}$ & $\mathrm{Ca}$ & $\mathrm{Mg}$ \\
\hline & & \multicolumn{6}{|c|}{ Massa de matéria seca } \\
\hline & & \multicolumn{6}{|c|}{ Raiz $\left(\mathrm{g} \mathrm{kg}^{-1}\right)$} \\
\hline \multirow{2}{*}{ Soja } & $\mathrm{SDH}$ & $25,10 \mathrm{a}$ & $4,43 \mathrm{a}^{*}$ & $27,11 \mathrm{ab}^{*}$ & $5,24 \mathrm{a}$ & $7,96 \mathrm{a}$ & $3,80 \mathrm{a}$ \\
\hline & $\mathrm{CDH}$ & $24,05 \mathrm{~A}$ & $3,15 \mathrm{~A}$ & $19,92 \mathrm{C}$ & $3,69 \mathrm{~A}$ & $8,18 \mathrm{~A}$ & $3,29 \mathrm{AB}$ \\
\hline \multirow{2}{*}{ Soja +} & $\mathrm{SDH}$ & $14,00 \mathrm{a}$ & $3,79 \mathrm{a}$ & $20,95 \mathrm{~b}$ & $3,00 \mathrm{ab}$ & $8,78 \mathrm{a}$ & $4,13 \mathrm{a}$ \\
\hline & $\mathrm{CDH}$ & $26,45 \mathrm{~A}$ & $3,03 \mathrm{~A}$ & $21,20 \mathrm{~B}$ & $4,06 \mathrm{~A}$ & $8,51 \mathrm{~A}$ & $9,09 \mathrm{~A}$ \\
\hline \multirow{2}{*}{ T. procumbens +} & $\mathrm{SDH}$ & $7,00 \mathrm{~b}$ & $1,91 \mathrm{~b}$ & $30,71 \mathrm{a}$ & $2,59 \mathrm{~b}$ & $10,43 \mathrm{a}$ & $4,69 \mathrm{a}^{*}$ \\
\hline & $\mathrm{CDH}$ & $5,70 \mathrm{~B}$ & $3,41 \mathrm{~A}^{*}$ & $32,25 \mathrm{~A}$ & $4,35 \mathrm{~A}^{*}$ & $7,68 \mathrm{~A}$ & $3,72 \mathrm{AB}$ \\
\hline \multirow{2}{*}{ T. procumbens } & $\mathrm{SDH}$ & $12,25 \mathrm{~b}$ & $1,39 \mathrm{~b}$ & $25,06 \mathrm{ab}$ & $3,53 \mathrm{ab}$ & $9,88 \mathrm{a}$ & $1,01 \mathrm{~b}$ \\
\hline & $\mathrm{CDH}$ & $7,75 \mathrm{~B}$ & $2,09 \mathrm{~B}^{*}$ & $24,55 \mathrm{~B}$ & $3,47 \mathrm{~A}$ & $7,93 \mathrm{~A}$ & $1,06 \mathrm{~B}$ \\
\hline & & \multicolumn{6}{|c|}{ Caule $\left(\mathrm{g} \mathrm{kg}^{-1}\right)$} \\
\hline \multirow{2}{*}{ Soja } & $\mathrm{SDH}$ & $15,38 \mathrm{a}$ & $2,98 \mathrm{a}$ & $33,79 \mathrm{~b}$ & $0,87 \mathrm{ab}$ & $6,86 \mathrm{~b}$ & $0,63 \mathrm{~b}$ \\
\hline & $\mathrm{CDH}$ & $17,74 \mathrm{~A}$ & $3,07 \mathrm{~A}$ & $38,93 \mathrm{~B}$ & $1,26 \mathrm{~B}$ & $8,77 \mathrm{C}^{*}$ & $3,72 \mathrm{~B}^{*}$ \\
\hline \multirow{2}{*}{ Soja +} & $\mathrm{SDH}$ & $17,32 \mathrm{a}$ & $3,10 \mathrm{a}$ & $36,87 \mathrm{~b}$ & $1,23 \mathrm{a}$ & $7,96 \mathrm{~b}$ & $1,41 \mathrm{~b}$ \\
\hline & $\mathrm{CDH}$ & $16,66 \mathrm{~A}$ & $2,71 \mathrm{~A}$ & $36,87 \mathrm{~B}$ & $1,06 \mathrm{~B}$ & $7,68 \mathrm{C}$ & $1,79 \mathrm{~B}$ \\
\hline \multirow{2}{*}{ T. procumbens +} & $\mathrm{SDH}$ & $11,65 \mathrm{~b}$ & $3,00 \mathrm{a}$ & $75,91 \mathrm{a}^{*}$ & $1,26 \mathrm{a}$ & $7,14 \mathrm{~b}$ & $3,62 \mathrm{ab}$ \\
\hline & $\mathrm{CDH}$ & $12,40 \mathrm{~B}$ & $2,48 \mathrm{~A}$ & $41,50 \mathrm{~B}$ & $2,78 \mathrm{~A}^{*}$ & $20,58 \mathrm{~A}^{*}$ & $8,15 \mathrm{~A}^{*}$ \\
\hline \multirow{2}{*}{ T. procumbens } & $\mathrm{SDH}$ & $11,51 \mathrm{~b}$ & $2,47 \mathrm{~b}$ & $75,91 \mathrm{a}$ & $0,56 \mathrm{~b}$ & $15,64 \mathrm{a}$ & $6,88 \mathrm{a}$ \\
\hline & $\mathrm{CDH}$ & $8,34 \mathrm{~B}$ & $1,89 \mathrm{~B}$ & $69,75 \mathrm{~A}$ & $0,68 \mathrm{~B}$ & $11,80 \mathrm{~B}$ & $3,93 \mathrm{~B}$ \\
\hline & & \multicolumn{6}{|c|}{ Folha $\left(\mathrm{g} \mathrm{kg}^{-1}\right)$} \\
\hline \multirow{2}{*}{ Soja } & $\mathrm{SDH}$ & $53,70 \mathrm{~b}^{*}$ & $3,15 \mathrm{ab}$ & $32,25 \mathrm{~b}$ & $2,33 \mathrm{a}$ & $9,06 \mathrm{~b}$ & $5,81 \mathrm{~b}$ \\
\hline & $\mathrm{CDH}$ & $48,74 \mathrm{~A}$ & $3,21 \mathrm{~A}$ & $37,39 \mathrm{~B}$ & $2,33 \mathrm{AB}$ & $10,70 \mathrm{~B}$ & $4,15 \mathrm{~B}$ \\
\hline \multirow{2}{*}{ Soja +} & $\mathrm{SDH}$ & $76,05 \mathrm{a}^{*}$ & $3,57 \mathrm{a}$ & $37,39 \mathrm{ab}$ & $2,42 \mathrm{a}$ & $10,98 \mathrm{~b}$ & $3,80 \mathrm{~b}$ \\
\hline & $\mathrm{CDH}$ & $39,81 \mathrm{~A}$ & $2,85 \mathrm{~A}$ & $32,25 \mathrm{~B}$ & $3,08 \mathrm{~A}$ & $10,91 \mathrm{~B}$ & $5,25 \mathrm{~B}$ \\
\hline \multirow{2}{*}{ T. procumbens +} & $\mathrm{SDH}$ & $28,56 \mathrm{c}^{*}$ & $2,28 \mathrm{~b}$ & $43,04 \mathrm{a}$ & $3,01 \mathrm{a}^{*}$ & $20,03 \mathrm{ab}$ & $7,31 \mathrm{~b}$ \\
\hline & $\mathrm{CDH}$ & $14,11 \mathrm{~B}$ & $3,11 \mathrm{~A}^{*}$ & $74,37 \mathrm{~A}^{*}$ & $1,37 \mathrm{~B}$ & $11,25 \mathrm{AB}$ & $5,22 \mathrm{~B}$ \\
\hline \multirow{2}{*}{ T. procumbens } & $\mathrm{SDH}$ & $28,47 \mathrm{c}$ & $2,51 \mathrm{ab}$ & $44,58 \mathrm{a}$ & $2,47 \mathrm{a}$ & $24,15 \mathrm{a}$ & $10,91 \mathrm{a}$ \\
\hline & $\mathrm{CDH}$ & $21,10 \mathrm{~B}$ & $1,93 \mathrm{~B}$ & $42,01 \mathrm{~B}$ & $2,33 \mathrm{AB}$ & $26,89 \mathrm{~A}$ & $10,07 \mathrm{~A}$ \\
\hline & & \multicolumn{6}{|c|}{ Total $\left(\mathrm{mg} \mathrm{planta}^{-1}\right)$} \\
\hline \multirow{2}{*}{ Soja } & $\mathrm{SDH}$ & $394,50 \mathrm{a}$ & $42,41 \mathrm{a}^{*}$ & $372,30 \mathrm{a}$ & $34,61 \mathrm{a}$ & $96,16 \mathrm{a}$ & $44,01 \mathrm{a}$ \\
\hline & $\mathrm{CDH}$ & $269,49 \mathrm{~A}$ & $26,68 \mathrm{~A}$ & $271,34 \mathrm{~A}$ & $20,93 \mathrm{~A}$ & $78,95 \mathrm{~A}$ & $32,36 \mathrm{~A}$ \\
\hline \multirow{2}{*}{ Soja +} & $\mathrm{SDH}$ & $557,06 \mathrm{a}^{*}$ & $42,38 \mathrm{a}^{*}$ & $429,83 \mathrm{a}^{*}$ & $25,30 \mathrm{a}$ & $117,56 \mathrm{a}$ & $36,25 \mathrm{a}$ \\
\hline & $\mathrm{CDH}$ & $251,80 \mathrm{~A}$ & $24,77 \mathrm{~A}$ & $284,42 \mathrm{~A}$ & $21,23 \mathrm{~A}$ & $81,44 \mathrm{~A}$ & $38,82 \mathrm{~A}$ \\
\hline \multirow{2}{*}{ T. procumbens +} & $\mathrm{SDH}$ & $47,69 \mathrm{~b}$ & $7,17 \mathrm{~b}$ & $162,03 \mathrm{~b}$ & $5,52 \mathrm{~b}$ & $33,21 \mathrm{~b}$ & $13,86 \mathrm{~b}$ \\
\hline & $\mathrm{CDH}$ & $49,17 \mathrm{~B}$ & $6,54 \mathrm{~B}$ & $125,01 \mathrm{~A}$ & $5,35 \mathrm{~B}$ & $35,41 \mathrm{~B}$ & $15,04 \mathrm{~B}$ \\
\hline \multirow{2}{*}{ T. procumbens } & SDH & $95,27 \mathrm{~b}$ & $13,32 \mathrm{~b}$ & $333,17 \mathrm{a}$ & $8,45 \mathrm{~b}$ & $98,22 \mathrm{~b}$ & $41,12 \mathrm{a}^{*}$ \\
\hline & $\mathrm{CDH}$ & $48,99 \mathrm{~B}$ & $6,93 \mathrm{~B}$ & $194,97 \mathrm{~A}$ & $6,05 \mathrm{~B}$ & $53,15 \mathrm{~B}$ & $22,02 \mathrm{~B}$ \\
\hline
\end{tabular}

1/ SDH - sem deficiência hídrica; CDH - com defíciência hídrica temporária, entre 20 e 40 dias após o transplante (DAT). Médias seguidas por mesma letra minúscula (comparação entre tratamentos $\mathrm{SDH}$ ) e maiúscula (comparação entre tratamentos $\mathrm{CDH}$ ) na coluna não diferem entre si pelo teste de Tukey $(\mathrm{p} \leq 0,05)$. * Diferença significativa $(\mathrm{p} \leq 0,05)$ pelo teste $\mathrm{t}$, entre os tratamentos $\mathrm{SDH}$ e $\mathrm{CDH}, \mathrm{para}$ a mesma composição de plantas. 
cultura, embora não seja possivel diferenciar quantitativamente quanto desse efeito é atribuído ao processo competitivo (parte aérea e radicular) e quanto representa a $\mathrm{DH}$.

O maior potencial competitivo de soja em relação a $T$. procumbens ficou evidenciado nas avaliações. Contudo, mesmo sob competição e restrição hídrica, a planta daninha apresentou florescimento e frutificação em todos os tratamentos avaliados, o que, aliado a sua capacidade de reprodução caulinar e à facilidade de distribuição anemófila das sementes, permite rápida proliferação dessa espécie nas áreas de cultivo.

\section{LITERATURA CITADA}

BAUMANN, D. T.; BASTIAANS, L.; KROPFF, M. J. Competition and crop performance in a leek-celery intercropping system. Crop Sci., v. 41, n. 3, p. 764-774, 2001.

BOSU, P. P.; WAGNER, M. R. Effects of induced water stress on leaf trachoma density and foliar nutrients of three elm (ulmus) species: implications for resistance to the elm leaf beetle. Environ. Entomol., v. 36, n. 3, p. 595-601, 2007.

BROUWER, R. Functional equilibrium: sense or nonsense? Nether. J. Agric. Sci., v. 31, n. 4, p. 335-348, 1983.

CHRISTOFFOLETI, P. J. Análise comparativa do crescimento de biótipos de picão-preto (Bidens pilosa) resistente e susceptível aos herbicidas inibidores de ALS Planta Daninha, v. 19, n. 1, p. 75-84, 2001.

COUSENS, R.; O'NEILL, M. Density dependence of replacement series experiments. Oikos, v. 66, n. 2, p. 347-352, 1993.

ERASMO, E. A. L.; BIANCO, S.; PITELLI, R. A. Estudo sobre o crescimento de fedegoso. Planta Daninha, v. 15, n. 2, p. 170-179, 1997.

FENG, Y. L.; CAO, K. F.; ZHANG, J. L. Photosynthetic characteristics, dark respiration, and leaf mass per unit area in seedlings of four tropical species grown under three irradiances. Photosynthetica, v. 42, n. 3, p. 431-437, 2004.

FENG, Y. L.; WANG, J.; SANG, W. Biomass allocation, morphology and photosynthesis of invasive and noninvasive exotic species grown at four irradiance levels. Acta Oecol. v. 31, n. 1, p. 40-47, 2007.

GONZÁLEZ, E. M. Solute heterogeneity and osmotic adjustment in different leaf structures of semi-leafless pea (Pisum sativum L.) subjected to water stress. Plant Biol., v. 4, n. 5, p. 558-566, 2002.
GOWING, D. J. G.; DAVIES, W. J.; JONES, H. G. A positive root-sourced signal as an indicator of soil drying in apple, Malus x domestica Borkh. J. Exper. Bot., v. 41, n. 233, p. $1535-1540,1990$.

GUIMARÃES, S. C.; SOUZA, I. F.; PINHO, E. V. R. V. Viabilidade de sementes de erva-de-touro, sob diferentes condições de armazenamento. Planta Daninha, v. 22, n. 2 , p. 231-238, 2004.

JEYABAL, A.; PALANIAPPAN, S. P.; CHELLIAH, S. Efficacy of metribuzin and trifluralin on weed management in soybean (Glycine max). Indian J. Agron., v. 46, n. 2, p. 339-342, 2001.

JURAIMI, A. S.; DRENNAN, D. S. H.; ANUAR, N. The effects of shading on the growth, development and partitioning of biomass in bermudagrass (Cynodon dactylon (L.) Pers. J. Biol. Sci., v. 4, n. 6, p. 756-762, 2004.

KUMUDINI, S.; HUME, D. J.; CHU, G. Genetic improvement in short season soybeans: i. dry matter accumulation, partitioning, and leaf area duration. Crop Sci., v. 41, n. 2 , p. $391-398,2001$

MALAVOLTA, E. et al. Avaliação do estado nutricional das plantas: princípios e aplicações. 2.ed. Piracicaba: Potafos, 1997. 319 p.

MOHAMMED, S. P.; et al. Clinical evaluation of Tridax procumbens leaf paste in the treatment of septic wound in cattle. Indian J. Field Veter., v. 3, n. 4, p. 43-45, 2008.

MORTENSEN, D. A.; BASTIAANS, L.; SATTIN, M. The role of ecology in the development of weed management systems: an outlook. Weed Res., v. 40, n. 1, p. 49-62, 2000.

NICHOLAS, J. C.; HARPER, J. E.; HAGEMAN, R. H Nitrate reductase activity in soybeans (Glycine max (L.) Merrill) 1- Effects of light and temperature. Plant Physiol., v. 58, n. 6 , p. $731-735,1976$.

OLIVEIRA, M. A. J. Atividade da redutase de nitrato em mudas de pupunheira (Bactris gasipaes) Ci. Rural, v. 35, n. 3 , p. $515-522,2005$.

PATTERSON, D. T. Shading responses of purple and yellow nutsedges (Cyperus rotundus and C. esculentus). Weed Sci., v. 30, n. 1, p. 25-30, 1982.

PEREIRA-NETTO, A. B.; MAGALHÃES, A. C. N.; PINTO, H. S. Nitrate reductase activity in field-grown Pueraria lobata (Kudzu) in Southeastern Brazil. Pesq. Agropec. Bras., v. 33, n. 12, p. 1971-1975, 1998.

PLHAK, F. Nitrogen supply through transpiration mass flow can limit nitrogen nutrition of plants. Plant Soil Environ., v. 49, n. 10 , p. $473-479,2003$. 
SHARP, R. E. Interaction with ethylene: changing views on the role of abscisic acid in root and shoot growth responses to water stress. Plant Cell Environ., v. 25, n. 2, p. 211-222, 2002 .
SINGH, D. A. P.; KUMAR, P.; SARAVANAKUMAR, V. R. Production performance of white giant rabbits fed with Tridax procumbens. Indian Veter. J., v. 84, n. 11, p. 1212-1213, 2007. 